Digilec 6 (2019), pp. 14-26

Fecha de recepción: 25/06/2019

Fecha de aceptación: 28/02/2020

DOI: https://doi.org/10.17979/digilec.2019.6.0.5425

(cc) BY-SA

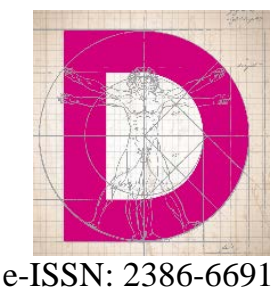

\title{
ELEMENTOS QUE INTERVIENEN EN EL ÉXITO -Y EN EL FRACASO- DE LA INTERACCIÓN ORAL EN LENGUA EXTRANJERA EN SECUNDARIA
}

\section{ELEMENTS WHICH INTERVENE IN THE SUCCESS -AND FAILURE- OF THE SPOKEN INTERACTION IN THE SUBJECT OF FOREIGN LANGUAGE IN SECONDARY}

\author{
Zeus PLASENCIA CARBALLO* \\ Universidad de La Laguna
}

\begin{abstract}
Resumen
A lo largo de las últimas décadas, la Didáctica de las Lenguas Extranjeras (DLE) ha gozado de un gran interés por parte de la esfera académica y profesional, indagando cómo mejorar el proceso de enseñanza-aprendizaje e implementar alternativas que permitan al docente cumplir con su rol de guía, y asistir a su alumnado durante dicho proceso. No obstante, de los cuatro modos de comunicación -Common European Framework of References for Languages. Companion Volume with New Descriptors- que fundamentan la enseñanza de las LE (recepción, producción, interacción y mediación), la interacción oral recibe una menor atención por parte del docente y ofrece resultados poco alentadores. Por esta razón, a lo largo de este artículo se ofrecerán algunas de las razones por las que esto ocurre, en especial en la etapa de Secundaria, además de sugerir una serie de alternativas que faciliten el siempre arduo proceso de hablar e interactuar en un código distinto al materno.
\end{abstract}

Palabras clave: Interacción oral; Secundaria; Lenguas extranjeras; docencia

\begin{abstract}
In the last decades, Foreign Language Teaching (FLT) has been the centre of great interest among academics and professionals concerned about the ways to improve both the language teaching methodologies and the role of teachers in guiding and assisting students' learning processes. Nevertheless, among the four modes of communication Common European Framework of References for Languages. Companion Volume with New Descriptors- underpinning the teaching of foreign languages, the spoken interaction has traditionally been the most neglected one. This paper explores some of the reasons that account for this situation in secondary education and presents ideas and alternatives to help students interact orally in the foreign language.
\end{abstract}

Key Words: Oral interaction; Secondary; Foreign languages; Teaching

*Fac. de Humanidades. Campus de Guajara, La Laguna (S. C. de Tenerife). Email: zplasenc@ull.edu.es. 


\section{INTRODUCCIÓN}

En el momento presente es incuestionable la importancia que rodea al hecho de hablar una o varias lenguas extranjeras (LE). La sociedad globalizada y globalizadora se encarga de convertir la capacidad de comunicarse por medio de distintos códigos lingüísticos en un requisito indispensable para el acceso a la información y al conocimiento que, en muchos casos, se topa con la propia lengua materna como frontera infranqueable.

Atendiendo a esto último, los organismos correspondientes a la esfera educativa muestran una constante preocupación por incentivar y mejorar la capacidad comunicativa del alumnado en otras lenguas por medio de planes y proyectos, como el Plan Europa 2020, los programas AICLE e inmersión lingüística, los programas para el impulso de las lenguas extranjeras a nivel autonómico, etc. No obstante, la presunción de que esta iniciativa a favor de las capacidades lingüísticas de los aprendices de lenguas extranjeras es efectiva y fructífera, puede no corresponderse, en muchos casos, con la realidad silenciada tras las puertas de las aulas de Secundaria. De hecho, la Encuesta Europea de Competencias Lingüísticas sitúa al alumnado de la etapa de Secundaria español en las posiciones más bajas, con respecto a la competencia lingüística de estos en la lengua inglesa (Hornero, Mur \& Plo, 2013: 111).

El enfoque comunicativo actual procura, sin duda alguna, el ejercicio oral activo e interactivo entre los aprendices de LE; sin embargo, tal y como apuntan GarcíaSampedro, Miranda-Morais, \& Iñesta-Mena (2018: 136), "podría decirse que la enseñanza de la lengua oral no forma parte de nuestra tradición escolar, lo que explicaría, en cierto modo, las carencias actuales en este campo". Esta incoherencia existente entre la consciencia teórica de lo que se debe hacer dentro de un aula de LE y la puesta en práctica de dichos saberes, entre otros, es uno de los principales objetivos sobre el que este artículo pretende ahondar, explicar y hacer reflexionar al enseñante. En virtud de esto último, desgranar los principales factores y circunstancias que intervienen, de un modo u otro, en el éxito o en el fracaso de la comunicación oral en lenguas extranjeras en las aulas de Secundaria será el elemento vertebrador a lo largo de las próximas líneas. De igual manera, se persigue hacer reflexionar al lector sobre aquellos elementos que obstaculizan un enfoque comunicativo oral adecuado dentro del aula, ofreciendo simultáneamente alternativas que ayuden al docente a abordar aquellas dificultades que puedan surgir dentro de su aula de LE al llevar a cabo actividades de naturaleza oral e interactiva.

\section{EL AULA DE LENGUA EXTRANJERA EN SECUNDARIA}

Son múltiples y variados los factores -y la naturaleza de estos- que acentúan la complejidad de la enseñanza en la etapa de Secundaria, tanto para el alumnado como para el docente. De entre ellos, se ha de destacar el propio cambio de etapa -de Primaria a Secundaria- más que evidente, en muchas ocasiones, durante el primer curso 
correspondiente al primer ciclo de Secundaria (1. ${ }^{\circ}$ ESO). En otro orden, se encuentra el período de transición hacia la madurez tanto física como psíquica conocido como pubertad, ligado, de manera indiscutible, a las relaciones socio-afectivas que establece el alumnado con sus iguales y con su entorno más inmediato. En esta línea, no se puede obviar que la parte afectiva de un individuo es inherente a su parte cognitiva (Schumann, 1994). Estos aspectos, entre otros, influyen y condicionan tanto la actividad docente como el proceso de enseñanza-aprendizaje dentro de las aulas de Secundaria. Además, y sin ánimo de generalizar, según Plasencia (2018), entre el alumnado de esta etapa se advierte un bajo interés y popularidad por el aprendizaje de lenguas extranjeras, factor que no ayuda a favorecer su enseñanza dentro de las aulas. Por tanto, atendiendo a todos estos indicadores, se evidencia que el esfuerzo humano, siempre respaldado por el entramado curricular, ha de ser constante y certero con el fin de sortear los impedimentos naturales correspondientes a la etapa y a la materia de LE.

Por otro lado, la generalidad es considerar el aula de LE como:

[...] un escenario social y cultural, y que el discurso que en ella se genera, a partir de la interacción entre los miembros que la componen, constituye un mecanismo esencial para entender cómo se realiza el proceso de negociación, construcción y representación de significados sobre los contenidos y tareas que se abordan en la actividad del aula (Salaberri, 2002: 71).

Dicho de otro modo, el aula de LE es el espacio donde se establecen las bases primarias para que el alumnado adquiera las herramientas necesarias (léxico, estructuras, estrategias, etc.) para poder entablar una acción comunicativa efectiva en la Lengua Extranjera. No obstante, este hecho no se ha de confundir con el perfeccionamiento de la lengua meta, el cual se debe complementar fuera del aula, en un entorno idóneo y contextualizado que sea capaz de cubrir todos aquellos aspectos que el aula ordinaria de LE no llega a alcanzar.

\section{HABLAR E INTERACTUAR EN UNA LENGUA EXTRANJERA}

\subsection{El currículo de LE}

Previamente, antes de aventurarse a la discusión sobre las destrezas de expresión e interacción oral y de qué modo se practican en el aula, es preciso conocer de manera sintética los elementos que integra el currículo y que, en cierto modo, mapean la compleja geografía de la materia de LE.

La Ley Orgánica para la Mejora de la Calidad Educativa (LOMCE) (2013), por la que se rige el currículo de lenguas extranjeras en la etapa de Secundaria, toma como referencia el Marco Común Europeo de Referencia para las Lenguas (MCERL) (2001), y sus consideraciones al respecto de las actividades de la lengua -oír, hablar, leer y escribir ${ }^{1}$ - para estructurar, de este modo, el actual currículo de LE. Como se puede apreciar, del

\footnotetext{
${ }^{1}$ Desde el año 2018 existe una actualización del MCERL (Common European Framework of References for Languages. Companion Volume with New Descriptors) que describe cuatro modos de comunicación: recepción, producción, interacción y mediación.
} 
concepto a la ley, todos y cada uno de los elementos se nutren los unos de los otros para confluir en un sistema organizado donde cada elemento desempeña una función y motiva la dinámica del siguiente. De este modo, el currículo establece sus bases sobre cinco grandes bloques de conocimiento que compilan las actividades o destrezas básicas de la lengua, junto con los aspectos sociolingüísticos, culturales y emocionales inherentes al aprendizaje de cualquier código extranjero. Igualmente, cada destreza tiene asignada uno o varios criterios de evaluación ${ }^{2}$, elementos centrales dentro del currículo a los que se les asocia un número de estándares de aprendizaje evaluables, cuya función es delimitar aquello que el alumnado conoce y puede hacer en la LE. Del mismo modo, el currículo de LE tiene a bien considerar la promoción de las competencias clave ${ }^{3}$ para favorecer su adquisición por medio de los criterios de evaluación y los estándares de aprendizaje.

Pese a la vasta complejidad que muestra el currículo de LE, la intencionalidad de estas líneas es focalizar la atención sobre el bloque II de conocimiento, asociado a las destrezas de la producción oral, tal y como se hará en el siguiente apartado.

\subsection{Producción vs. interacción}

Atendiendo a lo anterior, es necesario aclarar que las destrezas relacionadas con el bloque II de conocimiento (hablar) son dos: expresión oral e interacción oral. En muchos casos, debido al desconocimiento del alcance de estas destrezas, se tiende a incluir la interacción oral dentro de la expresión oral como una extensión de esta o, en el peor de los escenarios, a ignorar su necesidad de práctica.

Tanto la expresión como la interacción comparten un origen oral, como su propio nombre indica. Estas se caracterizan por ser manifestaciones de tipo breve y efímeras en el tiempo que nacen de la espontaneidad del agente -o agentes-implicado. Además, estas producciones orales poseen la cualidad de ser claras y directas, sin una complejidad extrema en su estructura textual, y enmarcadas en un espacio, un tiempo y unas circunstancias precisas para que el interlocutor llegue a comprender el mensaje emitido por el hablante. A pesar de ello, también es posible evidenciar diferencias notables entre ambas destrezas. Por ejemplo, mientras que la expresión oral, a priori, consiste en la transmisión individual de un mensaje a otro interlocutor o audiencia, la interacción implica a un mínimo de dos agentes o interlocutores que conversan, aunando características tanto de la destreza de expresión oral como de la comprensión oral, es decir, la interacción oral consiste en la transmisión de un mensaje, la escucha y comprensión -entendimiento- de dicho mensaje y, por último, la respuesta o réplica de este por parte del otro interlocutor participante. Oliver \& Philp (2014) plantean la interacción oral de la siguiente manera:

La interacción oral es la lengua hablada que tiene lugar entre dos o más personas y que, tal y como indica su nombre, es el tipo de producción oral y comprensión oral que se produce durante los intercambios comunicativos en tiempo real. [...] la

\footnotetext{
${ }^{2}$ Describen aquello que se quiere valorar y son el referente específico para evaluar el aprendizaje que el alumnado debe lograr en cada materia.

${ }^{3}$ Habilidades, actitudes, conocimientos y experiencias que faciliten un desarrollo eficaz, funcional y pleno de los individuos en la sociedad a lo largo de su vida.
} 
interacción oral es colaborativa y muy a menudo recíproca, donde cada interlocutor trabaja para co-construir un intercambio significativo ${ }^{4}$ (2014: 5).

En otras palabras, se podría afirmar que la destreza de expresión oral es una actividad activo-pasiva, donde el emisor ostenta el rol de activo y el receptor de pasivo; mientras que la interacción establece una relación activa constante entre los interlocutores implicados.

\subsection{Dos destrezas, tres criterios}

Tal y como se ha indicado con anterioridad, estas dos actividades de la lengua o destrezas se encuadran dentro del bloque II de conocimiento en el currículo de Secundaria. Del mismo modo, ambas destrezas están asociadas a tres criterios de evaluación: i) El criterio 3, relacionado con la destreza motor-perceptiva. Según Bygate, este tipo de destreza implica "percibir, recordar y articular en el orden correcto sonidos y estructuras de la lengua. Este es el aspecto relativamente superficial de la destreza” (Bygate, 1987: 5). Por tanto, estas son producciones nomológicas o presentaciones donde el alumno ha de "producir textos orales breves o de longitud media, adecuados al receptor y al contexto” (Decreto 83/2016, p. 18300). ii) El criterio 4, asociado a la destreza de interacción, donde "la destreza de interacción involucra la toma de decisiones sobre la comunicación, tal como: qué decir, cómo decirlo y si desarrollarlo de acuerdo con las intenciones de uno” (Bygate, 1987: 5). Así, este criterio atiende a la actividad oral interactiva, donde se requiere que el alumno sea capaz de "interactuar de manera sencilla pero efectiva en intercambios orales claramente estructurados, adecuando el registro al interlocutor y al contexto” (Decreto 83/2016: 18302). Y, por último, iii) el criterio 5, conexo a las estrategias relacionadas con ambas destrezas, donde el alumno ha de "aplicar las estrategias más adecuadas para elaborar producciones orales nomológicas o dialógicas” (Decreto 83/2016: 18304).

Tras evidenciar los aspectos más relevantes del currículo y cómo estos se interrelacionan con las destrezas de producción oral (expresión e interacción), se puede apreciar que el documento tiene en consideración todas y cada una de las particularidades de estas actividades de la lengua, y así lo refleja en su redacción. Sin embargo, en el siguiente apartado (4. La interacción en el aula de LE) se plantea si las especificaciones teóricas del currículo de LE se aplican debidamente en la práctica, en cuanto a la actividad de la interacción oral en el aula de Secundaria se refiere.

\section{LA INTERACCIÓN EN EL AULA DE LENGUA EXTRANJERA}

Considerando lo anteriormente expuesto en el apartado dedicado al currículo y las destrezas de naturaleza oral, la interacción oral se constituye como una destreza singular e indispensable dentro de la función comunicativa que cubren las lenguas extranjeras. A

\footnotetext{
${ }^{4}$ Traducción propia de "Oral interaction is the spoken language that takes place between two or more people and, as the name implies, is in the type of speaking and listening that occurs in real time in communicative exchanges. [...] oral interaction is collaborative and most often reciprocal, with each speaker working to co-construct a meaningful exchange".
} 
pesar de esta relevancia y necesidad dentro del proceso de enseñanza-aprendizaje, la interacción oral es una de las destrezas menos practicadas dentro del aula de LE, hecho que denota una contradicción palpable entre la teoría (currículo) y la práctica (ejercicio docente) en el aula. En esta línea, Hornero, Mur, \& Plo (2013), a partir de su estudio, plantean que muchos estudiantes coinciden en el ejercicio constante de la gramática y el vocabulario (Hornero, Mur, \& Plo, 2013: 115), recibiendo las destrezas orales cada vez menos empuje por parte de los docentes de LE, y disminuyendo a medida que los alumnos avanzan a lo largo de su etapa escolar (Hornero, Mur, \& Plo, 2013: 121).

Previo al abordaje de los inconvenientes con los que se topa la destreza de interacción oral en lengua extranjera en las aulas de Secundaria (4.2.), es pertinente conocer las implicaciones que conlleva el aula de LE dentro del proceso de enseñanzaaprendizaje de las lenguas. Citando a Escobar y Nussbaum (2002: 37), "la interacción cara a cara es el medio y lugar privilegiado en el que el alumno se apropia de la lengua”. Las autoras, tal y como refleja la cita, consideran que el aula de LE debe ser un espacio con un clima adecuado y acogedor que motive la libertad de participación y expresión por parte del alumnado en el código extranjero. Únicamente de este modo, el alumnado podrá “apropiarse de la lengua” y llevar a cabo el fin último para el que el aula se erige: comunicar en la lengua meta.

Otro de los pilares fundamentales en el aula de LE es la interacción implícita entre los agentes educativos (docentes y discentes), quienes, en primera instancia, hacen uso de la lengua -en algunas ocasiones la lengua meta, en otras la lengua materna ${ }^{5}$ - con un fin didáctico:

[...] los procesos de enseñanza y aprendizaje son situaciones interactivas y comunicativas en las que los educadores ayudan a los alumnos de forma sistemática, bien sea planificada o no, a construir conocimiento y el habla es uno de los medios más poderosos a través del cual se ejerce esa ayuda (Saleberri, 2002: 71).

Por este motivo, la producción, y en especial la interacción, se manifiestan imprescindibles dentro del aula en cualquier fase del proceso de enseñanza-aprendizaje.

\subsection{Tipos de interacción}

Sin lugar a equívoco, tal y como se adelantaba en el punto anterior, dentro del aula de LE se identifican dos agentes educativos principales: el docente y los discentes. Estos agentes, a su vez, y durante el ejercicio del acto comunicativo oral, pueden entablar dos tipos de relaciones de interacción oral: i) la interacción profesor-alumno y ii) la interacción alumno-alumno.

La primera de ellas se contrapone al habitual speech del docente, donde el alumno asume un papel pasivo dentro de la conversación y se limita a intervenir de manera puntual. De este modo, se ha de entender la interacción profesor-alumno como un tipo de conversación fluida entre ambos agentes en la lengua meta, donde el aporte dialógico sea equilibrado y coherente en esencia. No obstante, el factor fluidez al que se alude, en

\footnotetext{
${ }^{5}$ El propio currículo entiende la lengua materna como apoyo lingüístico que facilite el proceso de aprendizaje de un código extranjero. Es, por ende, un elemento asistencial y no de rechazo dentro del aula de LE.
} 
ciertos casos, se muestra inestable -incluso etéreo- dentro del aula de LE, debido a las barreras o muros previamente levantados entre ambos agentes: profesor y alumno. Estos muros tienen un origen, principalmente, instrumental y emocional. El primero se refiere al nivel de destreza gramatical, léxico y sintáctico que posee el alumnado en la lengua extranjera; es decir, cuán eficientes son los aprendices a la hora de emplear dichos elementos lingüísticos durante el desarrollo de una situación comunicativa. Por otro lado, el segundo se adentra más en el aspecto psicológico del alumnado, quien, atendiendo al período vital con el que coincide su paso por la etapa de Secundaria (pubertad), puede y suele presentar algún tipo de inseguridad, fobia o miedo a la hora de enfrentarse a la interacción oral en la lengua extranjera con otro interlocutor, en este caso con el docente. Este último factor está directamente relacionado con la hipótesis del filtro afectivo de Stephen Krashen, quien considera que un ambiente de aprendizaje incómodo, poco distendido e inseguro promueve un filtro afectivo alto, es decir, un obstáculo entre el aprendiz y la adquisición de la lengua meta, mientras que una atmósfera agradable, distendida y que denote seguridad favorece un filtro afectivo bajo y, por ende, menor dificultad para que el aprendiz adquiera la lengua extranjera. Además, otra posible razón, tal y como apuntan Arévalo-Balboa \& Briesmaster (2018), parafraseando a Brown (2001): “[...] muchos estudiantes no se sienten preparados para hablar en la lengua meta, lo que hace que el desarrollo de las destrezas orales en el alumnado sea un reto de grandes proporciones” ${ }^{6}$ (2018: 144).

Por tanto, considerando las variables que rodean a la interacción oral profesoralumno mencionadas, es prioritario que el aula se convierta en un lugar de comunicación segura, donde el alumnado disponga de la libertad suficiente para realizar incursiones comunicativas orales con el docente, derribando o sorteando las barreras emocionales que puedan surgir entre ambos. Este objetivo, a priori cuasi idílico, se fundamenta a partir de la simple premisa de invitar al alumno a hablar. En apartados consecutivos se puntualizará cómo propiciar esta invitación a la conversación.

El segundo tipo de interacción (alumno-alumno) también tiene lugar en un escenario no menos complejo que la comunicación profesor-alumno ya descrita. Autores como Escobar y Nusbaum (2002); Arnó (2002) o García (2006), coinciden en los beneficios que ofrece este tipo de conversaciones en el plano emocional (aumento de la seguridad y descenso de la ansiedad en los interlocutores) y en el plano académico (negociación del significado, modificación del input, etc.). Plasencia (2018) considera que "la interacción entre iguales se presume más natural -orgánica- que la propia interacción con el docente, a quien el alumnado suele relacionar constantemente con un agente evaluador y/o punitivo, o que la misma producción monológica del alumnado” (2018: 132-133).

No obstante, este tipo de interacción también alberga luces y sombras, tal y como se mencionaba anteriormente: en primer lugar, se ha de mencionar la complejidad que entraña el hecho de que los aprendices puedan establecer una conversación coherente y fluida en la lengua meta de manera autónoma; en segundo lugar, las limitaciones de

\footnotetext{
${ }^{6}$ Traducción propia de “[...] many students do not feel ready to produce the target language, which makes developing oral skills in students a challenge of major proportion”.
} 
carácter instrumental (léxico y gramática) y de carácter emocional (inseguridades, vergüenza...) pueden llegar a obstaculizar e inhibir cualquier intento conversación en la lengua meta entre iguales; en tercer lugar, mantener conversaciones cuyo eje temático sea ajeno a la realidad o al interés del alumnado tendrá más probabilidad de obstaculizar y estancar la conversación que si fuese un tema de interés y cercano a los interlocutores; por último, en cuarto lugar, las interacciones pautadas o guionizadas, como por ejemplo los role plays, inherentemente implican "la anulación implícita -total o parcial- de la espontaneidad que puedan desarrollar los interlocutores durante el acto comunicativo oral” (Plasencia, 2018: 136).

\subsection{Obstáculos}

Al margen de la potencialidad que ofrece el aula-clase como entorno privilegiado para la enseñanza y el aprendizaje de las lenguas extranjeras, tal y como se aduce en líneas anteriores, además de las virtudes y defectos que presentan los distintos tipos de interacción que toman lugar en el aula, existen numerosos obstáculos que impiden un adecuado desarrollo del proceso de enseñanza y aprendizaje de las lenguas. Plasencia (2018), por su parte, enumera los siguientes:

[...] De entre ellos, el más evidente y recurrente es la preparación previa del alumnado, es decir, los conocimientos que estos ya poseen sobre la lengua meta. Si bien es cierto que la enseñanza del inglés en España es preponderante desde etapas tempranas de escolarización, el estudio de esta lengua acusa de un grave raquitismo en secundaria, debiéndose este último a que se le ha inculcado al alumnado -desde una edad temprana- que la materia de Inglés se basa meramente en el aprendizaje de léxico y estructuras gramaticales de manera continua y casi exclusiva. A tenor de esto último, es más que comprensible la impopularidad resultante hacia las lenguas extranjeras y el miedo que surge entre el alumnado que llega a los ciclos de la ESO (2018: 120).

Así pues, se puede inferir que, en la actualidad, todavía existe la prevalencia de un tipo de enseñanza de la LE basada en el modelo tradicional, donde el vocabulario y la gramática se "entrenan" de manera exhaustiva y continuada por medio de ejercicios destinados a dicho fin, junto al predominio del ejercicio de las destrezas de expresión escrita y comprensión tanto oral como lectora sobre las destrezas orales. Con respecto a esto último, Pavón (2006) apunta que “aun cuando debe existir una enseñanza equilibrada entre las destrezas escritas y las orales, estas últimas quedan relegadas normalmente a un papel testimonial con respecto a las destrezas de carácter escrito” (2006: 420). Las razones por las que acontece lo señalado por Pavón (2006) son múltiples y variadas; no obstante, Plasencia (2018) lista cinco causas principales:

i) El carácter poco lucido de la destreza, atendiendo a lo efímero de esta y la complejidad que presenta para su atesoramiento (a excepción de que sea recopilada por medio de una grabación), en comparación con el testimonio físico que lega la expresión escrita.

ii) La temporalización, puesto que la gestión de los grupos y el cumplimiento de las programaciones de aula y de los vastos currículos juegan en contra del desarrollo de las destrezas orales (Plo, Hornero \& Mur, 2014: 61). Las 
destrezas orales, en palabras de Plasencia (2018), requieren "tiempo, esfuerzo y voluntad por ambas partes, tanto por el docente como por el alumnado" (2018: 121).

iii) La evaluación de la destreza que, por lo general, se suele realizar del mismo modo que la expresión oral, sin incidir en las singularidades que presenta. Escobar y Nussbaum (2002) inciden en este aspecto, donde "no es frecuente que las escalas de evaluación incorporen aspectos propiamente interactivos” (2002: 46).

iv) Las características individuales de los interlocutores, donde salen a la luz las capacidades, actitudes y aptitudes de cada uno de los agentes comunicativos.

v) La autoconfianza ${ }^{7}$ de estos integrantes durante el acto interactivo oral (Plasencia, 2018: 121-123).

\subsection{Alternativas}

La descripción de la interacción oral en las aulas de LE de Secundaria presentada no resulta muy halagüeña. No obstante, uno de los principales cometidos de todo docente es identificar los problemas que acontecen en el aula y tratar de buscar soluciones o alternativas a los mismos. Con este espíritu, a continuación, se ofrece una serie de orientaciones dirigidas al docente, las cuales surgen como alternativas referidas a los inconvenientes señalados con anterioridad.

Aludiendo nuevamente a la hipótesis del filtro afectivo de Stephen Krashen (1982), en un primer momento, la prioridad para el docente ha de ser la configuración de un ambiente de aprendizaje seguro donde prime la confianza entre todos los agentes educativos (alumnado y profesorado). De este modo, la ansiedad ${ }^{8}$ derivada de la interacción oral -con el docente o entre iguales- en la lengua meta se verá sustancialmente reducida, pues se entiende que el aula de LE es un ambiente seguro donde el error es motivo de aprendizaje y no de reproche o burla; de esta manera, el filtro afectivo desciende y se favorece la promoción de la expresión oral en la lengua meta.

Junto al espacio y ambiente adecuados para propiciar la comunicación, también se debe tener en consideración el tiempo que cada alumno necesita para familiarizarse con los elementos tanto lingüísticos como paralingüísticos en la LE. Esto último se puede favorecer "entrenando" al alumnado en incursiones orales breves y pausadas, donde surja la interacción de manera natural, sin forzar y tratando de mantener la comodidad entre los agentes involucrados. Casamassima \& Insua (2015), a este respecto, afirman: “a más inglés hablen en clase nuestros alumnos, más oportunidades tienen para mejorar su

\footnotetext{
${ }^{7}$ En referencia a la inseguridad, vergüenza o temor durante el acto comunicativo oral, llegando a ser un impedimento o barrera de índole emocional que impida la comunicación entre interlocutores en la lengua extranjera.

${ }^{8}$ Horwitz et al. (1986) y Arnáiz-Castro et al. (2013, 2016a, 2016b) enumeran muchas razones y/o factores que desencadenan en ansiedad en los aprendices de LE.
} 
desempeño en inglés y, como resultado, se espera que lo hagan mejor en los exámenes orales" ${ }^{9}$ (2015: 22).

Al igual que es prioritario entrenar al alumnado en la práctica de la destreza interactiva oral, es igualmente necesario preparar al alumnado a aceptar el error a la hora de hablar y a entenderlo como parte intrínseca durante el aprendizaje de cualquier lengua extranjera, pues solo se aprende a hablar hablando (Rabèa, 2010: 14).

Un entorno que favorece la participación es importante, pero no es suficiente para invitar al alumno a hablar. A tenor de esto último, es imperante proponer temas de interés general o particular, teniendo en cuenta la intención comunicativa que se desee durante la interacción. Además, la conciencia sobre los contextos e intereses del alumnado facilita en gran medida captar la atención de este, pues se activa un elemento fundamental para la participación: la motivación. A este respecto, Dörnyei (2005) sostiene que el factor motivación activa, en un primer momento, el interés inicial para que se lleve a cabo el aprendizaje de la LE y, a posteriori, la razón por la que mantener el a veces duro proceso de aprendizaje del código extranjero (2005: 65).

En último lugar, se plantea una de las cuestiones más conflictivas y difíciles de abordar dentro del aula de LE en la etapa de Secundaria: la continuidad en el uso del código extranjero por parte de los interlocutores. Este aspecto, que en un primer momento puede entenderse como arbitrario por parte de los agentes que toman parte en la interacción, está intrínsecamente relacionado, entre otros, con el nivel instrumental en la lengua meta que posee el alumnado, con el aspecto emocional (estrés y ansiedad) e inclusive con la práctica en esta destreza. Si bien es cierto que el aprendiz realiza un esfuerzo evidente en la comunicación con el docente -debido, posiblemente, a la diferencia de roles y estatus de cada uno- durante la comunicación entre iguales este esfuerzo se diluye, posiblemente a causa de que el docente no arbitra el acto comunicativo-interactivo en todo momento y ello, indudablemente, abre camino hacia un cambio de código por parte de los participantes. A este respecto, el docente debe propiciar un compromiso entre ambas partes (alumnado y docente), que se puede reflejar a través de una ficha de seguimiento gestionada por el propio alumnado que, al mismo tiempo, le sirva de herramienta para la reflexión sobre qué, cuánto y cómo han hablado en la lengua extranjera.

Del mismo modo, la presencia de grabadoras o dispositivos que graben el acto comunicativo, tal y como sugieren Escobar y Nussbaum (2002), “ejerce en la pareja funciones de regulación y de control de la actividad” (2002: 41). Esta alternativa desempeña, en cierto modo, una función punitiva sobre los agentes que interactúan; sin embargo, se muestra menos imponente que la presencia continuada del docente durante el desarrollo de la comunicación entre iguales, tal y como apunta Plasencia (2018: 140).

Plo et al (2014) sintetizan las alternativas expuestas de la siguiente manera:

Necesitamos ser conscientes de que el desarrollo del discurso oral solo puede ocurrir con el paso del tiempo y requiere de una aproximación consistente por parte del docente para contextualizar el trabajo en el aula, proporcionar oportunidades para

\footnotetext{
${ }^{9}$ Traducción propia de "the more our students speak English in class, the more chances they have to improve their performance in English, and as a result, they are expected to perform better in oral exams".
} 
interactuar, y ofrecer una evaluación por ambas partes del docente y del estudiante ${ }^{10}$ (29).

En otras palabras, es indispensable que el docente lleve a cabo una organización previa y a conciencia de sus sesiones de LE, donde los contenidos, el contexto y el aspecto motivacional, junto con la práctica continuada de la comunicación oral en el aula, deriven en el hábito de la interacción en el código extranjero.

\section{CONCLUSIONES}

De acuerdo con García (2006): “el aprendizaje de una lengua se entiende como una práctica interactiva, colaborativa y socialmente contextualizada” (2006: 558). Para que dicho aprendizaje tenga lugar y, además, logre activar el aspecto colaborativo y social dentro del aula de LE, es primordial ofrecer un ambiente propicio y seguro para el aprendizaje de la LE, donde el alumnado se sienta cómodo y motivado para llevar a cabo una interacción comunicativa con cualquier agente (docente o discentes) dentro del aula. Es un hecho que el docente no puede controlar todos y cada uno de los aspectos involucrados en la comunicación en la lengua extranjera dentro del aula; sin embargo, conocer el potencial y las limitaciones del alumnado, y sus intereses y motivaciones, favorece una aproximación al objetivo que todo docente tiene a la hora de enseñar dicha lengua: que su alumnado sea capaz de comunicarse en la lengua meta.

El planteamiento de la realidad descrita a lo largo de estas páginas no es sencillo. Aun así, es imperante que los docentes de LE tomen conciencia -a través de la investigación en acción en el aula- de qué funciona y qué no dentro en sus clases, y lograr sortear los continuos obstáculos que surgen durante la enseñanza y el ejercicio de esta parte tan central dentro de la enseñanza de las lenguas que supone la producción e interacción oral en la lengua meta.

Por último, rescatando la idea de sociedad cambiante e interconectada del inicio, y atendiendo a la actual migración hacia el aula 3.0, no se puede obviar el hecho de que las Tecnologías para la Información y Comunicación (TIC) se postulan como las grandes aliadas para la docencia del hoy y del mañana a este lado del planeta. A pesar de que este artículo no tiene como objetivo profundizar en la aplicabilidad de las TIC dentro del desarrollo de las destrezas orales en el aula de LE, sí que reconoce que este es un nicho de investigación y de aplicación bastante nutrido del que podrían beneficiarse las alternativas propuestas en el texto (García-Sánchez, 2016: 152).

\section{REFERENCIAS BIBLIOGRÁFICAS}

Arévalo-Balboa, F. \& Briesmaster, M. (2018). “Claim - Support - Question” Routine to Foster Coherence within Interactive Oral Communication among EFL Students. Profile: Issues in Teachers' Professional Development, vol. 20 (pp. 143-160).

\footnotetext{
${ }^{10}$ Traducción propia de "We do need to be aware that the development of oral discourse can occur only over time and requires a consistent approach by the teacher to contextualize work in the classroom, provide opportunities for interaction, and offer assessment on the part of both the teacher and the students".
} 
Bogotá. Recuperado

de:

https://revistas.unal.edu.co/index.php/profile/article/view/63554

Arnáiz-Castro, P. \& Guillén, F. (2013). Anxiety in Spanish EFL students in different university degree programmes. Anales de Psicología, 29 (2), 335-344.

Arnáiz-Castro, P. \& Pérez-Luzardo, J. (2016a). A Study on the correlation between anxiety and academic self-concept in interpreter trainees. Círculo de Lingüística Aplicada a la Comunicación, 67, 57-88.

Arnáiz-Castro, P. \& Pérez-Luzardo, J. (2016b). The teacher as a source of anxiety for university students. En A. Díez, V. Brotons, D. Escandell, J. Rovira (Eds.), Aprendizajes Plurilingües y Literarios. Nuevos Enfoques Didácticos (pp. 812-818). Alicante: Universidad de Alicante.

Arnó, E. (2002). El desarrollo de las habilidades metalingüísticas a través del trabajo en grupo. En J. Cots \& L. Nussbaum (Eds.), Pensar lo dicho. La reflexión sobre la lengua y la comunicación en el aprendizaje de lenguas (pp. 87-100). Lleida: Milenio.

Bygate, M. (1987). Speaking. Oxford: Oxford University Press.

Casamassima, M., \& Insua, F. (2015). On how thinking shapes speaking: Techniques to enhance students’ oral discourse. English Teaching Forum, 53 (2), 21-29.

Consejo de Europa (2018). Common European Framework of References for Languages. Companion Volume with New Descriptors. Recuperado de: https://rm.coe.int/cefrcompanion-volume-with-new-descriptors-2018/1680787989

Decreto 83/2016, de 4 de julio, por el que se establece el currículo de la Educación Secundaria Obligatoria y el Bachillerato en la Comunidad Autónoma de Canarias. Boletín Oficial de Canarias, núm. 136, de 4 de julio de 2016, 17046-19333. Recuperado de http://www.gobcan.es/boc/2015/169/002.html

Dörnyei, Z. (2005). The Psychology of Language Learner: Individual differences in Second Language Acquisition. Mahvah, NJ: Lawrence Erlbaum.

Escobar, C., \& Nussbaum, L. (2002). ¿Es posible evaluar la interacción oral en el aula?. En L. Miquel \& N. Sans (Coord.), Didáctica del español como lengua extranjera (pp. 37-52). Madrid: Fundación Actilibre.

García, M. (2006). La interacción oral alumno/alumno en el aula de lenguas extranjeras: líneas de investigación y consideraciones didácticas. En Las destrezas orales en la enseñanza del español L2-LE: XVII Congreso Internacional de la Asociación del Español como lengua extranjera (ASELE) (pp. 555-566). Logroño: Universidad de La Rioja.

García Sampedro, M., Miranda Morais, M. \& Iñesta Mena, E. (2018). Comunicación oral y m-learning en el aula de inglés de primaria: la fotografía y el vídeo como recurso. Fonseca, Journal of Communication, 16, 135-154. doi:10.14201/fjc201816135154

García-Sánchez, S. (2016). Encouraging collaborative interaction in EFL learners with video role-plays. ENSAYOS, Revista de la Facultad de Educación de Albacete, 31 (1). Recuperado de: http://www.revista.uclm.es/index.php/ensayos

Hornero, A.M., Mur, P. \& Plo, R. (2013). Oral Skills in the Spotlight: EFL in Secondary Education in a Spanish Local Context. Synergy, 9 (2), 111-124. Recuperado de: 
http://synergy.ase.ro/issues/2013-vol9-no-2/06-ana-maria-hornero-pilar-murduenas-ramon- plooral-skills-in-the-spotlight-efl-in-secondary-education-in-aspanish-local-context.pdf

Horwitz, E. K., Horwitz, M. B., \& Cope, J. (1986). Foreign language classroom anxiety. The Modern Language Journal, 70(2), 125-132.

Krashen, S. D. (1981). Second Language Acquisition and Second Language Learning. Oxford: Pergamon Press.

Krashen, S. D. (1982). Principles and Practice in Second Language Acquisition. Oxford: Pergamon Press.

Pavón, V. (2006). Análisis de las causas por las que la enseñanza de las destrezas orales obtiene resultados insatisfactorios en el entorno del aula y propuesta de mejora. En M. Amengual, M. Juan-Garau, J. Salazar (Coords.), Adquisición y aprendizaje de lenguas en contextos plurilingües. Ensayos y propuestas aplicadas (pp. 417-431). Palma de Mallorca: Edicions UIB.

Plasencia, Z. (2018). La interacción oral en el aula de Lengua Extranjera en Secundaria: estrategias, actividades y procesos de aprendizaje (tesis doctoral no publicada). Santa Cruz de Tenerife: Universidad de La Laguna.

Plasencia, Z. (2018). Oral production in the Foreign Language classroom: Teachers, Reflections and Suggestions. International Journal of Anglisticum. Literature, Linguistics \& Interdisciplinary Studies, 7 (6), 10-15.

Plo, R., Hornero, A.M. \& Mur, P. (2014). Implementing the teaching/learning of oral skills in secondary education in Aragón: gauging teachers' attitudes, beliefs and expectations. International Journal of English Studies, 14 (1), 55-77. doi: 10.6018/ijes/14/1/175041

Rabéa, B. (2010). El desarrollo de la expresión oral en lengua extranjera. En I Simposio de Didáctica del Español (pp. 9-14). Argel: Instituto Cervantes de Argel. Recuperado de https://cvc.cervantes.es/ensenanza/biblioteca_ele/publicaciones_centros/PDF/arge 1_2010/02_rabea.pdf

Oliver, R. \& Philp, J. (2014). Focus on Oral Interaction. Oxford: Oxford University Press.

Salaberri, M.S. (2002). El discurso de los docentes en el aula como mediador en la reflexión sobre la lengua. En J. Cots, L. Nussbaum (Eds.), Pensar lo dicho. La reflexión sobre la lengua y la comunicación en el aprendizaje de lenguas (pp. 7185). Lleida: Milenio.

Schumann, J. (1994). Where is cognition? Emotion and cognition in second language learning. Studies in second language acquisition, 16, 231-242. 